\title{
Remarks about Immunosenescence
}

\section{PhD. Zamir Calamita*}

Doctor and Specialist in Allergies and Immunology, Head of the Discipline of Allergies and Immunopathology, Marília Medical School (FAMEMA) and Tenured Professor of the Postgraduate Group on Health and Aging, FAMEMA, São Paulo, Brazil

*Corresponding Author: Zamir Calamita, Doctor and Specialist in Allergies and Immunology, Head of the Discipline of Allergies and Immunopathology, Marília Medical School (FAMEMA) and Tenured Professor of the Postgraduate Group on Health and Aging, FAMEMA, São Paulo, Brazil.

\section{Received: March 16, 2020}

Published: April 24, 2020

(C) All rights are reserved by Zamir Calamita.

\begin{abstract}
This is a mini-review addressing the main aspects of immunosenescence, including: predisposition towards infections, inflammation, autoimmunity, cancer, response to vaccines, allergy, stress, nutrition and physical activity. Remarks are made about this complex network of possible alterations relating to aging.

Keywords: Allergy, Autoimmunity; Aging; Immunosenescence; Infection; Neoplasia; Vaccine
\end{abstract}

\section{Introduction}

Mean life expectancy has increased greatly over recent decades. Aging has thus become an important focus of studies in many sectors, especially within the field of healthcare [1]. One of the main fields of study within the medical sciences involved with senescence relates to the immunological system, which undergoes a variety of changes consequent to aging, with alterations to the responses to infections and vaccines, occurrences of states of chronic inflammation, repercussions regarding the autoimmune response and increased incidence of neoplasia, among other changes [2].

\section{Immunosenescence}

The set of alterations to the immune system that are observed as consequences of aging is known as immunosenescence. This is often linked to progressive decline in immunological function, which mainly consists of deficient responses to combating infections and to vaccines, impaired performance of immunological vigilance relating to neoplasia and alterations to autoimmunity and allergic processes. Immunosenescence may also be associated with nutritional and emotional disorders, among other possible repercussions [2-12].

In addition, through aging, the profile of interleukins (IL) that are released tends to change towards those with pro-inflammatory action, i.e. interferon (INF) gamma, tumor necrosis factor (TNF)alpha and IL-6. Thus, there is a tendency for atherosclerotic vascular diseases and dementia to arise [8,13].

Over the course of life, the immunological system is continually exposed to stimuli and morphological and functional changes. Its functioning reaches its peak level at puberty, with a gradual decline thereafter, with aging, in parallel with involution of the thymus [2-9]. The immunological system is closely related to several other sectors of the organism, including the individual's mental, neurological, endocrinological and nutritional situation. Homeostasis is required for controlling the immune response, so as to impede excessive responses or auto- aggression. This control is provided mainly by cells of the regulatory T cell class (Treg) [2-11].

Regarding primary lymphoid tissues, at bone marrow level, the number of stem cells and the capacity for cell self-renewal are seen to decrease with aging. This has a direct repercussion on hematopoiesis. In parallel, colony-stimulating factors diminish and this, in association with involution of the thymus, has repercussions for lymphocyte maturation. This limitation to cell proliferation potential is linked to erosion of telomers, which are chromatin structures that cap and protect the ends of chromosomes. Regeneration of telomers depends on telomerase activity, which declines with age $[5,6]$.

The thymus is responsible for "educating" T lymphocytes (TL), which orchestrate the immune response, through positive and negative selection of specific antigens. Atrophy of the thymus occurs through aging, such that by the age of 50 years, 5 to $10 \%$ of the thymus mass remains. The epithelial cells of the thymus produce large numbers of colony-stimulating factors, such as the interleukins IL-1, IL-3 and IL-7 (this last one is essential for lymphocytes). On the other hand, there is also increased production of cytokines that induce atrophy of the thymus, such as IL- 6 and LIF (leukemia inhibitory factor) [2-9].

The immune response is didactically divided into the innate response (natural) and the adaptive response (acquired). These two responses often present a correlation and both of them can become altered through aging. The innate or natural response is the first to act against aggressors, and it consists mainly of the following: a- mechanical barriers provided by the skin and mucosa, with their secretions; b- macrophages and neutrophils, which are cells with high phagocytic capacity that do not have a memory; c - natural killer (NK) cells, which are mainly implicated in the response to viruses and in vigilance against tumor cells; d- pattern recognition receptors such as toll-like receptors (TLR), which are present 
in a variety of cells and especially in those of the immune system, and which recognize the pathogen-associated molecular patterns (PAMPs) that are present in various infectious agents; and e- the complement system.

Macrophages also participate in the start of the adaptive or acquired response: after phagocytosis, they have the capacity to present antigenic structures from the phagocytized structure, through the major histocompatibility complex (MHC), to T lymphocytes. Thus, together with dendritic cells, macrophages belong to the class of so-called "antigen-presenting cells" (APC). They have the capacity to release a variety of fundamental interleukins, in triggering the organism's defense response. These include IL-1, IL-6, IL-8 and TNF.

Regarding the innate (natural) response, which is the organism's first line of defense, it has been observed that immunosenescence leads neutrophils to have diminished chemotactic, phagocytic and bactericidal capacity and to have lower superoxide production, along with greater tendency to undergo premature apoptosis, thus impeding long presence at the infection site. They also undergo diminution of the number of receptors such as CD16 and CD11 on their surface, which are essential for correct functioning of neutrophils [2-10]. In macrophages, alterations similar to those seen in neutrophils are seen, along with lower capacity for expression of MHC class II molecules. This therefore also has repercussions relating to elaboration of the adaptative immune response.

Regarding NK cells, although they do not decrease in number, their functional activity decreases such that they have lower capacity to respond to cytokines, diminished cell cytotoxicity and diminished interleukin and chemokine synthesis. This diminishes their response to viral infections and impairs their capacity for immunological surveillance against tumor cells.

Dendritic cells form the link between innate and adaptative immunity. Immunosenescence seems to lead them to secrete more IL-6 and TNF-alpha, but to fail to generate an anti- inflammatory response. This is the so-called paradox of increased inflammatory activity in elderly people ("inflammaging") [2-8].

In relation to the adaptative or acquired response, it is observed that immunosenescence may be accompanied by a cell or humoral response that has become insufficient. Despite some divergences of opinion, the current consensus is that the main alterations relate to $\mathrm{T}$ cell activity, i.e. the cell immune response [2-12]. No alteration to the total number of lymphocytes has been observed, but some qualitative alterations may be found. These include the following: a- reduction of the number of virgin $\mathrm{T}$ cells (CD45RA+) and increased numbers of memory clones (CD45RO); b- diminished proliferation of TL due to phytohemagglutinin; c- relative reduction of the number of CD4 lymphocytes in relation to CD8 lymphocytes; $\mathrm{d}$ - change to the relative proportionality of $\mathrm{T}$ helper lymphocytes, with an increased Th-2/Th-1 ratio cause by decline in IL-2 and e- reduction of the expression of CD154 surface molecules, which are important in cell activation of TL. Despite the increase in the number of memory T cells, they present lower expression of CD28 surface molecules, which interact with B7 molecules in APC and are fundamental for cell activation. Thus, they multiply less when exposed to antigens.

Regarding B lymphocytes, the possible effects of aging may include diminution of the numbers of co-stimulatory molecules such as the CD40 surface molecule, along with lower affinity for immunoglobulins (Ig), but higher production of plasmacytes and immunoglobulins that act insufficiently (deficient opsonization) [2-12].

Regarding cytokines, these can be divided didactically into proinflammatory cytokines, i.e. INF-gamma, TNF-alpha, IL-1, IL-2, IL6, IL-12 and IL-18; and anti-inflammatory cytokines, i.e. TGF-beta and IL-10, which are synthetized mainly by the family of Treg cells. With increased levels of the inflammatory cytokines IL-1, IL-6 and TNF-alpha, a chronic inflammatory state known as "inflammaging" arises. This is connected with increased levels of atherosclerosis and dementia at advanced ages [13].

Regarding vaccination, it has been observed that at advanced ages, the protective effect of immunization is diminished [2-16]. For example, in the case of the influenza virus, the efficacy of vaccination among healthy adults is 70 to $90 \%$, while among elderly people the efficacy is between 30 and $40 \%$. The degree to which protection is maintained is directly related to the intensity of the serum conversion and protection that is reached more than one month after the vaccination [15].

Another matter that has been much discussed in relation to aging is the question of auto- immunity [17]. Auto-tolerance can be defined as the capacity that the immunological system has for discerning between self and non-self. For this to occur, the organism makes use of the clonal selection process that begins in the thymus, where it is called central tolerance, and then extends to the peripheral level, i.e. peripheral tolerance. This is executed by Treg lymphocytes (CD4+, CD25+ and FOXP3). Conversely, the autoimmune process may be stimulated by some classes of cytokines such as IL-17. Thus, the imbalance between the actions of the interleukins produced by Treg cells (IL-10 and TGF-beta) and IL-17, favoring IL-17, may increase the predisposition towards development of autoimmune diseases [18]. In practice, higher prevalence of auto-antibodies is observed, but not always accompanied by autoimmune diseases.

In a recent study, increased levels of Treg cells were found in elderly people, but without statistical significance. The authors of that study suggested that further studies with larger samples should be conducted, focusing on Treg cells. One of the current hypotheses is that increased levels of Treg cells could be a physiological response that has the aim of countering possible autoimmune manifestations derived from higher prevalence of elevated levels of auto-antibodies in elderly people [10]. In an alternative hypothesis, increased levels of Treg cells could impair anti-tumor surveillance and the fight against infections through excessive containment of the immune response produced by these cells [10].

Another aspect of immunosenescence is the increasing incidence of neoplasia that is observed with aging [2-8,19-22]. A variety of factors may explain this higher predisposition towards neo- 
plasia, as follows: a- damage to DNA over the course of life caused by the great diversity of chemical, biological and physical aggressors to which the organism is exposed; b- the chronic inflammatory state ("inflammaging") that is often observed with aging; and c- genetic mutations leading to dysfunction of genes that suppress oncogenesis, such as $\mathrm{Rb}$ and $\mathrm{p} 53$, or leading to activation of genes that induce oncogenesis, such as myc and ras. These factors, together with those listed earlier, may give rise to impairment of anti-tumor immunosurveillance. This may occur both within the natural immunity through dysfunction of NK cells and within the acquired immunity through alterations to the function of CD4 and CD8 T cells (cell response) or reduced action by immunoglobulins against tumor antigens (humoral response).

Regarding nutritional issues within aging, the organism may undergo alterations relating to food intake and digestion and to absorption of some nutrients that are necessary for immunocompetence. There may be dysfunction of deglutition; the motility of the digestive tract tends to become slower; the taste buds become diminished; and secretion of saliva decreases. The diet may also be inadequate, which may give rise to nutritional disorders and secondary immunodeficiency relating to proteins, vitamins and trace elements (for example, deficiencies of vitamins $\mathrm{C}$ and $\mathrm{E}$ and zinc) $[3,23]$.

Another topic that has been addressed in some studies within geriatrics is allergies among elderly people [24-26]. Two situations have generally been highlighted: pruritus occurs more frequently in this age group, often related to the condition of these individuals' skin, which is drier [26-29] and elderly people present greater numbers of drug-related hypersensitivity reactions [27,28], because of their increasing exposure to situations of polypharmacy with advancing age. Elderly people become increasingly exposed to a variety of classes of drugs, which increases the chance of occurrence of drug-related adverse reactions.

Anotherissuethatis extremely importantis elderly people's emotional condition. A variety of conditions like depression and stress $[3,30]$ are often present at the end of their lives and these may have indirect implications such as loss of appetite or may have direct neuroendocrine-immunological implications. Indirectly, inadequate nutrient intake because of diminished appetite (which often accompanies depressive conditions) may have nutritional repercussions relating to the adequacy of the immunological response, as mentioned earlier. Moreover, in a direct manner, stressful situations run their course with elevation of cortisol and catecholamine levels. This leads to suppression of IL-12 production and disturbance of the balance of the Th1/Th2 ratio towards Th2, thereby diminishing the cell immune response [30].

Another topic, the importance of physical activity cannot be forgotten, especially among elderly people [5,31,32], while obviously respecting individuals' physical limitations. Elderly people who remain continually physically active present better immunological responses than those of sedentary elderly people. Aerobic physical activity diminishes "inflammaging" through lowering the levels of pro-inflammatory interleukins (IL-1, IL-6, INF-gamma and
TNF-alpha) and raising the levels of anti-inflammatory cytokines (TGF-beta and IL-10). It also have positive effects in terms of mood and favors good social integration, with favorable emotional repercussions and hence beneficial effects for the immunological system.

Lastly, a very current data concerns the pandemic by the coronavirus disease, whose lethality is higher in the elderly, where the median age was 64 years in severe cases, compared to 51.5 years in no severe cases $(\mathrm{P}<.001)$ [33]. Studies have shown that the disease caused by SARS- CoV-2 (severe acute respiratory syndrome coronavirus), recently named as COVID-19 (coronavirus disease 2019) by World Health Organization (WHO), could induce symptoms including fever, dry cough, dyspnea, fatigue, and lymphopenia in infected patients [33]. In more severe cases, infections causing viral pneumonia may lead to severe acute respiratory syndrome (SARS) and even death that occur mainly in the elderly probably due to the effects of immunosenescence.

\section{Conclusion}

Immunosenescence occurs concomitantly with complex remodeling of the entire organism, with modifications to many immunological parameters. To a certain extent, these can be regarded as physiological changes, but they may come accompanied by other situations that are deleterious for the individual's state of health. These other factors may include nutritional disorders, impaired emotional states and lack of physical activity. Advancing age is likely to be accompanied by health complications. Hence, humans' increasing life expectancy places studies on immunosenescence among the major priorities of science. It is important not only to have a long life but also to be able to reach the final stage of life with good health and quality of life. To achieve this, it is important to gain better understanding of the human immunological system.

\section{Bibliography}

1. Burch JB., et al. "Advances in geroscience: impact on healthspan and chronic disease". The Journals of Gerontology Series A Biological Sciences and Medical Sciences 69.1 (2014): S1-S3.

2. Gruver AL., et al. "Immunosenescence of ageing". The Journal of Pathology 211.2 (2007): 144-156.

3. Mota SMQ et al. "Imunossenescência: alterações imunológicas no idoso". RBM 67.6 (2010): 183-188.

4. Esquenazi DA., "Imunossenescência: as alterações do sistema imunológico provocadas pelo envelhecimento". Revista HUPE 6.1 (2008): 38-45.

5. Ewers I., et al. "Imunologia e envelhecimento". Einstein, São Paulo 6.1 (2008): S13-20.

6. Agondi RC., et al. "Imunossenescência". Revista Brasileira de Alergia e Imunopatologia 35.5 (2012): 169- 176.

7. Torres KCL., et al. "Imunossenescência”. Geriatria and Gerontologia 5.3 (2011): 163-169. 
8. Tonet AC. "Imunossenescência: a relação entre leucócitos, citocinas e doenças crônicas". Revista Brasileira de Geriatria e Gerontologia 11.2 (2008): 259-273.

9. Peres A., et al. "Imunossenescência - o envolvimento das células T no envelhecimento". Biociências, Porto Alegre 11.2 (2003): 187-194.

10. Lopes AB., et al. " Effects of immunosenescence on the lower expression. of surface molecules in neutrophils and lymphocytes". Current Aging Science 11 (2018): 118-125.

11. Dewan SK., et al. "Senescent remodeling of immune system and its contribuition to the predisposition of elderly to infections". Chinese Medical Journal 125.18 (2012): 3325- 3331.

12. Colonna-Romano G., et al. "B cells in aged: CD27, CD5, and CD40 expression". Mechanisms of Ageing and Development 124 (2003): 389-393.

13. Xia S., et al. "Na uodate on inflamm-aging: mechanisms, prevention, and treatment". Journal of Immunology Research (2016): ID8426874.

14. Oliveira FJ., et al. "Vacinação em idosos". Revista HUPE 6.1 (2007): 42-50.

15. Skowronski DM., et al. "Rapid decline of influenza vaccineinduced antibody in elderly: is it real, o ris it relevant?". The Journal of Infectious Diseases 195.15 (2008): 490-502.

16. Lang PO., et al. "Immunological pathogenesis of main age-related diseases and frailty: role of immunosenescence". European Geriatric Medicine 1 (2010): 112-121.

17. Vadasz Z., et al. "Age-related autoimmunity". BMC Medicine 11 (2013): 94.

18. Figueiredo AS., et al. "The T helper type $17 /$ regulatory T cell paradigm in pregnancy". Immunology 148 (2016): 13-21.

19. Rodrigues AFF. "Sistema imunológico no combate ao câncer: evasão da vigilância imunológica". Revista Facider 3.5 (2013).

20. Silva MM., et al. "Envelhecimento: importante fator de risco para o câncer”. Arquivos Médicos do ABC 30.1 (2005): 11-18.

21. Ahmad A., et al. "Aging and Inflammation: etiological cuprits of câncer". Current Aging Science 2.3 (2009): 174-186.

22. Foster AD., et al. "The aging imune system and its relationship with câncer”. Aging Health 7.5 (2011): 707-718.

23. Malafaia G. "As consequências da deficiências nutricionais associadas àimunossenescência na saúde do idoso". Arquivos Brasileiros De Ciências Da Saúde 33.3 (2008): 168-176.

24. Cardona V., et al. "Allergic diseases in the elderly". Clinical and Translational Allergy 1 (2011): 11.
25. Ventura MP., et al. "Incidence of allergic diseases in an elderly population". Immunopharmacology and Immunotoxicology 32.1 (2010): 165-170.

26. De Martins M., et al. "Allergy and aging: on old/new emerging health issue". Aging and Disease 8.2 (2017): 162-175.

27. Alves LDS., et al. "Sintomas de alergia nos idosos atendidos em um laborat'rio de geriatria”. Scientia Medica 24.3 (2014): 269273 .

28. Alves LDS., et al. "Estudo comparativo da prevalência de alergias entre idosos e não idosos". The Journal of Allergy and Clinical Immunology 2.2 (2014): 75-80.

29. Seyfarth F., et al. "Dry skin barrier function and irritant contact dermatites in the elderly". Clinics in Dermatology 29 (2011): 31-36.

30. Montoro J., et al. "Stress and allergy". Journal of Investigational Allergology and Clinical Immunology 19.1(2009): 40-47.

31. Pontes Jr FL., et al. "Atividade física no idoso: considerações gerais". The Revista Brasileira de Medicina 54.10 (1997): 840843.

32. Terra R., et al. "Efeito do exercício no sistema imune: resposta adaptação e sinalização celular". Revista Brasileira de Medicina do Esporte 18.3 (2012): 208-214.

33. Zhang J-J., et al. "Clinical characteristics of 140 patients infected with SARS-CoV-2 in Wuhan, China". Allergy 0.0 (2020): 1-12.

\section{Assets from publication with us}

- Prompt Acknowledgement after receiving the article

- Thorough Double blinded peer review

- Rapid Publication

- Issue of Publication Certificate

- High visibility of your Published work

Website: https://www.actascientific.com/

Submit Article: https://www.actascientific.com/submission.php Email us: editor@actascientific.com

Contact us: +919182824667 